\title{
A Method for Computing Heat Transfer Between Connected Compartments in a Zone Fire Model
}

Glenn P. Forney and William F. Moss

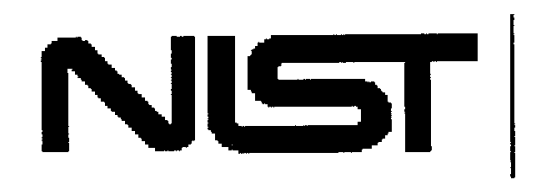

United States Department of Commerce Technology Administration

National Institute of Standards and Technology 


\section{A Method for Computing Heat Transfer Between Connected Compartments in a Zone Fire Model}

Glenn P. Forney

Building and Fire Research Laboratory

National Institute of Standards and Technology

William F. Moss

Department of Mathematical Sciences

Clemson University

July 1998

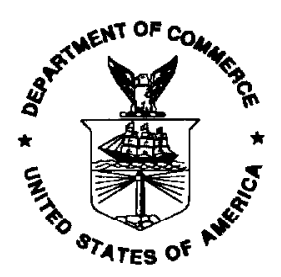

U.S. Department of Commerce

William M. Daley, Secretary

Technology Administration

Gary R. Bachula, Acting Under Secretary for Technology

National Institute of Standards and Technology

Raymond G. Kammer, Director 


\section{Contents}

1 Introduction 1

2 Conduction - Inter-compartment heat transfer 2

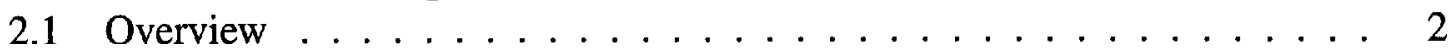

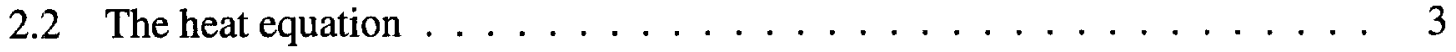

2.3 The discretized heat equation, the PR method . . . . . . . . . . . 4

3 Radiation - Heat transfer between walls divided into horizontal strips 6

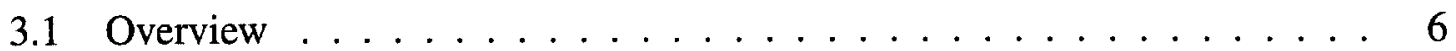

3.2 The Net Radiation Equation $\ldots \ldots \ldots$. . . . . . . . . 7

3.2.1 Configuration Factor Calculations ............ 9

3.2 .2 Transmissivities . . . . . . . . . . . . . . . 13

3.2 .3 Solid angles . . . . . . . . . . . . . 15

3.2 .4 Computing the source term, $c \ldots \ldots \ldots 17$

3.2.5 Radiant Heat Absorbed by Gas Layers . . . . . . . . . . . . . . . 18

3.3 Solution Properties . . . . . . . . . . . . . . 20

4 RADSTRIP0 - A simplified approach to computing radiation exchange 21

5 Summary $\quad 22$

$\begin{array}{ll}\text { References } & 23\end{array}$

$\begin{array}{ll}\text { Acknowledgements } & 24\end{array}$

A Implementation of an Inter-compartment heat transfer algorithm. 25

A.1 Data Structures . . . . . . . . . . . . . . . 25

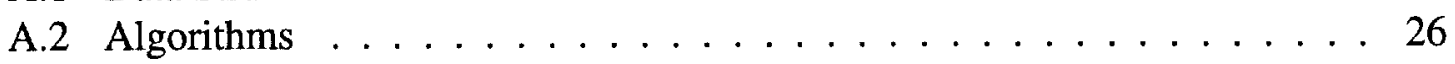

B Implementation $\quad 26$

B.1 Data Structures . . . . . . . . . . . . . . . 26

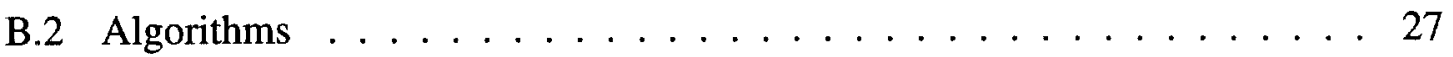




\section{List of Figures}

1 Computational Grid for the PR Method. . . . . . . . . . . . . 4

2 Setup for radiation heat transfer calculation in a room whose walls are divided into $\mathrm{M}$ horizontal strips. The faces or wall surfaces are labeled $u$, $f, r, b, l$ and $d$ for top, front, right, back, left and down respectively. The width, length and height of the compartment are given by $\mathrm{W}, \mathrm{L}$ and $\mathrm{H}$ respectively. The $i$ th strip from the top of the right face is given by $r_{i}$. Strips for other faces are indicated similarly. . . . . . . . . . 7

3 Input and Output Energy Distribution at the k'th Wall Surface $\ldots . . .8$

4 Configuration Factor Setup For Pairs of Parallel and Perpendicular Rectangles 9

5 Diagram illustrating the configuration factor identity, $A_{a b} F_{a-d}=A_{c d} F_{b-d}$, where $A_{a b}$ denotes the combined areas of surfaces a and $\mathrm{b}$ and $A_{c d}$ is defined analogously. . . . . . . . . . . . . . . . 11

6 Cross-sectional view of a compartment with wall surfaces $j$ and $k$. The path between $j$ and $k$ has length $L$. The portion of the path in the lower layer has length $L^{L}$ and the portion in the upper layer has length $L^{U} \ldots \ldots 13$

7 Solid Angle Setup For an Arbitrarily Oriented Triangle . . . . . . . . . 16

8 Solid Angle Setup For an Arbitrarily Oriented Triangle . . . . . . . . . . 16

9 Schematic illustrating energy deposited into the upper layer, the lower layer and arriving at the $k^{\prime} t h$ wall surface due to the $j^{\prime} t h$ wall surface. . . . . 18

10 Schematic illustrating energy deposited into the lower layer, into the upper layer, and arriving at the $k^{\prime} t h$ wall surface due to the $f^{\prime} t h$ fire. . . . . . 20 


\title{
A Method for Computing Heat Transfer Between Connected Compartments in a Zone Fire Model
}

\author{
Glenn P. Forney* William F. Moss ${ }^{\dagger}$
}

\begin{abstract}
This note describes a method for calculating conductive heat transfer between connected compartments in a zone fire model. The conduction problem is solved by dividing a compartment into six partitions corresponding to the floor and ceiling and the front, right, back and left walls. The wall partitions are further divided into several equally sized horizontal slabs or strips in order to approximate the vertical wall temperature profile. Radiative and convective flux boundary conditions are computed for the front and back surface of each partition/strip.

Some of the difficulties encountered when computing radiation exchange for large numbers of wall surfaces are alleviated by taking advantage of symmetry and the numerical characteristics of the linear system of equations that are solved. All strips have the same height. Strips on opposite walls have the same width. Strips do not change size with time. Using this information, the number of necessary configuration factor and transmissivity factors are reduced substantially as is the computing time for these factors. For wall surfaces that are nearly black,i.e. emissivities close to one, the system of equations to be solved (the net radiation equations) are strongly diagonally dominant. Therefore iterative algorithms (analogous to ray tracing) rather than direct methods such as Gaussian elimination can be used for determining the net radiative fluxes.
\end{abstract}

\section{Introduction}

This note describes the theory and an implementation plan for computing inter-compartment heat transfer in the zone fire model, CFAST[1]. Currently in CFAST 3.1.3, inter-compartment heat transfer only occurs through the floor/ceiling of vertically connected rooms; horizontal

\footnotetext{
${ }^{*}$ Building and Fire Research Laboratory, National Institute of Standards and Technology, Gaithersburg, MD 20899, U.S.A.

${ }^{\dagger}$ Department of Mathematical Sciences, Clemson University, Clcmson, SC 29634-1907, U.S.A.
} 
heat transfer (through vertical walls) only occurs to the outside. This report then documents a method for computing heat transfer between connected compartments in a zone fire model in both a horizontal and vertical direction.

Convective and radiative heat transfer terms serve as flux boundary conditions for the heat conduction problem. Convection, radiation and conduction algorithms, therefore, need to be designed consistently. Recent work[2] has demonstrated that by dividing a compartment partition into a small number of strips, it is possible to calculate heat transfer between connected compartments with sufficient accuracy. Unlike the upper and lower wall surfaces presently used in the model, these strips remain fixed in size throughout a simulation.

The present method of computing radiation transfer ${ }^{1}[3,4]$, is no longer suitable for modeling radiative heat transfer in a room with wall strips (unless the wall strip temperatures are lumped together to match the input that is expected by this present method) since the number of independent wall surfaces has increased. Two algorithms, RADSTRIP and RADSTRIP0, are then described for computing radiative heat transfer in a room with wall strips. RADSTRIP treats all strip temperatures independently. RADSTRIP0, a simplified version of RADSTRIP, lumps strips together and uses RAD4[4] (a four wall radiation model) to compute the radiative heat exchange.

\section{Conduction - Inter-compartment heat transfer}

\subsection{Overview}

Modeling inter-compartment heat transfer (ICHT) is analogous to modeling vent flow. Both models require data structures describing how walls or vents connect adjacent compartments. Both models compute energy flow between compartments; the ICHT model uses the heat equation while the vent flow model uses an integrated form of Bernoulli's law. The vent flow model also calculates mass transfer.

This section presents a method for modeling energy transfer between compartments using the heat equation. The fundamental modeling structure for this transfer will be a partition. A series of partitions are "assembled" to form a compartment. Each compartment is composed of six partitions corresponding to the front, right, back and left walls and the floor and ceiling. Each partition connects two compartments (one of the compartments may be the outside). Heat is transferred or conducted across a partition using the heat equation given the appropriate convective and radiative flux boundary conditions for each side of the partition.

In general, a three dimensional (3D), time dependent, heat conduction problem must be solved to model heat transfer in an arbitrary three dimensional structure. This is generally

\footnotetext{
${ }^{1}$ Radiation exchange is presently computed between four independent wall surfaces (floor, lower and upper walls and ceiling)
} 
not necessary in a zone fire model. Modeling the one dimensional (1D) temperature variation through ceiling and floors is sufficient to model heat transfer between floors. Modeling two dimensional (2D) temperature variation through a wall and vertically in a wall is sufficient to model heat transfer between compartments which share a common wall. Further, because the wall thickness is typically much smaller than the other two dimensions, vertical variation can be adequately modeled by dividing the wall into a number of horizontal strips and by solving a 1D heat conduction problem in each strip. This is referred to as the multiple 1D method. A full 2D solution is only needed for extremely thick walls. The work in [2] uses the Alternating Direction Method (ADI) of Peaceman and Rachford (PR) documented in reference [5] to handle both the multiple 1D and the 2D cases.

\subsection{The heat equation}

The equation for modeling heat flow in a wall with depth $W$ and height $Y$ (we assume that there is no temperature gradient along the width of the slab) can be written

$$
\begin{aligned}
\frac{\partial u}{\partial t}(x, y, t) & =\alpha\left(\frac{\partial^{2} u}{\partial x^{2}}(x, y, t)+\frac{\partial^{2} u}{\partial y^{2}}(x, y, t)\right) \\
-K \frac{d u}{d x}(0, y, t) & =q_{\text {conv }}^{\text {interior }}+q_{\text {rad }}^{\text {interior }} \\
-K \frac{d u}{d x}(W, y, t) & =q_{\text {conv }}^{\text {"exterior }}+q_{\text {rad }}^{\text {fexterior }} \\
\frac{d u}{d y}(x, 0, t) & =0 \\
\frac{d u}{d y}(x, Y, t) & =0 \\
0 & <x<W \\
0 & <y<Y \\
0 & <t .
\end{aligned}
$$

where $u$ is temperature, $\alpha=k /(\rho c)$ is thermal diffusivity, $k$ is thermal conductivity, $\rho$ is density and $c$ is specific heat and $x$ and $y$ are spatial coordinates within the wall and $t$ is time. Flux boundary conditions are used at the intcrior and exterior partition surfaces. Insulating boundary conditions are used at all other surfaces ${ }^{2}$.

To examine $2 \mathrm{D}$ vs 1D heat conduction, change variables in the above heat equation using $\hat{x}=x / W, \hat{y}=y / Y$ and $\hat{U}(\hat{x}, \hat{y}, t)=u(x, y, t)$ to obtain

$$
\frac{\partial \hat{U}}{\partial t}(\hat{x}, \hat{y}, t)=\alpha\left(\frac{1}{W^{2}} \frac{\partial^{2} \hat{U}}{\partial \hat{x}^{2}}(\hat{x}, \hat{y}, t)+\frac{1}{Y^{2}} \frac{\partial^{2} \hat{U}}{\partial \hat{y}^{2}}(\hat{x}, \hat{y}, t)\right)
$$

\footnotetext{
${ }^{2}$ The top and bottom surfaces of the partition.
} 


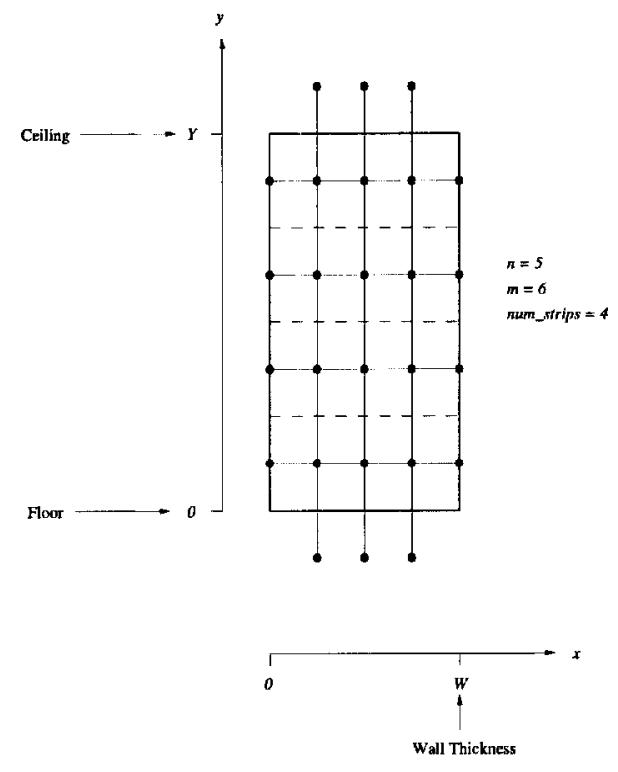

Figure 1: Computational Grid for the PR Method.

$$
\begin{aligned}
& =\hat{\alpha}\left(\frac{\partial^{2} \hat{U}}{\partial \hat{x}^{2}}(\hat{x}, \hat{y}, t)+\varepsilon \frac{\partial^{2} \hat{U}}{\partial \hat{y}^{2}}(\hat{x}, \hat{y}, t)\right) \\
0 & <\hat{x}<1 \\
0 & <\hat{y}<1 \\
0 & <t
\end{aligned}
$$

where $\varepsilon=(W / Y)^{2}$ and $\hat{\alpha}=\alpha / W^{2}$.

For thin wall materials typically found in fire scenarios it can be demonstrated that the ratio

$$
\frac{\varepsilon \frac{\partial^{2} \hat{U}}{\partial \hat{y}^{2}}(\hat{x}, \hat{y}, t)}{\frac{\partial^{2} \hat{U}}{\partial \hat{x}^{2}}(\hat{x}, \hat{y}, t)}<<1
$$

and therefore for most problems the multiple 1D method is adequate for modeling heat transfer between compartments which share a common wall.

\subsection{The discretized heat equation, the PR method}

Consider a 2D wall section with an $x$-axis through the wall and a $y$-axis from bottom to top as shown in Figure 1. Divide the inside surface $(x=0)$ of the wall into $k$ "real" horizontal strips of equal height $\Delta y$ and then add a "phantom" strip at the top and bottom of the wall. 
Starting at the center of each strip, define a horizontal line in the $x$-direction containing $n$ points with the last point on the outside surface of the wall. These points are closer together near the endpoints of the lines (wall surfaces) in order to obtain a more accurate approximation of the heat flux there. This yields $m=k+2$ horizontal lines in the $x$-direction, each containing $n$ points. This set of $n m$ points are vertices in an $x-y$ rectangular grid made up of $m$ lines in the $x$-direction and $n$ lines in the $y$-direction.

An outline of a time step of the PR method follows.

1. Starting at time $t$, advance to time $t+\Delta t / 2$ by solving a $1 \mathrm{D}$ heat conduction problem along each of $n-2$ interior vertical lines in the $y$-direction. At the ends of these vertical lines (top and bottom of the wall), apply implicit (time $t+\Delta t / 2$ ) boundary conditions. At the interior points of these vertical lines, use an implicit (time $t+\Delta t / 2$ ) centered difference approximation to $u_{y y}$, and use a time-lagged (time $t$ ) centered difference approximation to $u_{x x}$ using values already computed at time $t$. Use a forward difference approximation for $u_{t}$. Each resulting discretized heat equation (one heat equation for each vertical line) is tri-diagonal and uses an identical coefficient matrix. Therefore this matrix is factored only once and used to solve the heat conduction problem for each vertical line. At this point, $n-2,1 \mathrm{D}$ heat conduction problems with source terms have been solved.

2. Next, advance to time $t+\Delta t$ by solving a $1 \mathrm{D}$ heat conduction problem along the $k$ "real" horizontal lines in the $x$-direction (don't solve along the phantom lines). At the ends ( $x=0$ and $x=W$ ) apply convective and radiative boundary conditions. At the interior points along these lines, use a time-lagged (time $t+\Delta t / 2)$ centered difference approximation to $u_{y y}$, and use an implicit (time $t+\Delta t$ ) centered difference approximation to $u_{x x}$. Use a forward difference approximation for $u_{t}$. Again, each heat equation (this time in the horizontal direction) requires the solution of a tridiagonal system with the same coefficient matrix.

The PR method uses the following difference approximations to discretize equation (1). Let $U_{i, j, t}=u\left(x_{i}, y_{j}, t\right), \Delta x_{i}=x_{i+1}-x_{i}$, and $\Delta y=y_{j+1}-y_{j}$. Note that the spacing is uniform only in the $y$-direction and that each approximated second derivative contains three terms.

$$
\begin{aligned}
\frac{\partial^{2} u}{\partial x^{2}}\left(x_{i}, y_{j}, t\right) \approx U X X_{i, j, t} \equiv & \frac{2}{\Delta x_{i}\left(\Delta x_{i}+\Delta x_{i-1}\right)} U_{i-1, j, t}-\frac{2}{\Delta x_{i} \Delta x_{i-1}} U_{i, j, t} \\
& +\frac{2}{\Delta x_{i-1}\left(\Delta x_{i}+\Delta x_{i-1}\right)} U_{i+1, j, t} \\
\frac{\partial^{2} u}{\partial y^{2}}\left(x_{i}, y_{j}, t\right) \approx U Y Y_{i, j, t} \equiv & \frac{U_{i, j+1, t}-2 U_{i, j, t}+U_{i, j-1, t}}{\Delta y^{2}} .
\end{aligned}
$$

The PR equations for the 1D solutions obtained by discretizing the heat equation at the 
interior points along the vertical lines in the $y$-direction are

$$
\frac{U_{i, j, t+\Delta t / 2}-U_{i, j, t}}{\Delta t / 2}=\alpha\left[U X X_{i, j, t}+U Y Y_{i, j, t+\Delta t / 2}\right] .
$$

Equations for the boundary conditions at the endpoints of these lines must be included. For each $i$ in equation (17), a tridiagonal linear system is obtained. The PR equations for the $1 \mathrm{D}$ solutions obtained by discretizing the heat equation at the interior points along the horizontal lines in the $x$-direction are

$$
\frac{U_{i, j, t+\Delta t}-U_{i, j, t+\Delta t / 2}}{\Delta t / 2}=\alpha\left[U X X_{i, j, t+\Delta t}+U Y Y_{i, j, t+\Delta t / 2}\right]
$$

Equations for the boundary conditions at the endpoints of these lines must be included. For each $j$, a tridiagonal linear system is obtained. If the solutions in the $y$-direction are omitted and $\Delta t / 2$ is replaced by $\Delta t$, the PR method becomes the multiple 1D method.

\section{Radiation - Heat transfer between walls divided into horizontal strips}

\subsection{Overview}

Consider a compartment, illustrated in Figure 2, with a floor, ceiling and four side walls. Each side wall is divided into $M$ horizontal strips. This compartment then has $N=4 M+2$ wall surfaces. This section documents procedures for calculating the radiation exchange between these $N$ surfaces, two gas layers and a collection of point source fires. The computer time required to perform these calculations can easily dominate a zone fire modeling calculation if careful attention is not given to how they are performed. Two facts about this problem scenario can be exploited.

First, the proposed problem is highly symmetric. Opposite walls are parallel, joining walls are perpendicular. Strip sizes and locations are the same on opposite walls. Wall surface geometries are fixed in time. As a rcsult, it is shown how the required $(4 M+2)^{2}$ configuration factors can be computed algebraically (i.e. no expressions involving expensive to compute transcendental functions such as tan or ln, etc.) from a subset of $5 M+1$ configuration factors computed directly. Unlike the four-wall radiation model presented in $[3,4]$ the configuration factors here need only be computed once since the wall surface geometry is fixed in time.

Second, for wall surfaces that are nearly black $^{3}$, the linear systems arising from the net radiation equations are highly diagonally dominant. Iterative methods, which in this case

\footnotetext{
${ }^{3}$ emissivities close to one
} 


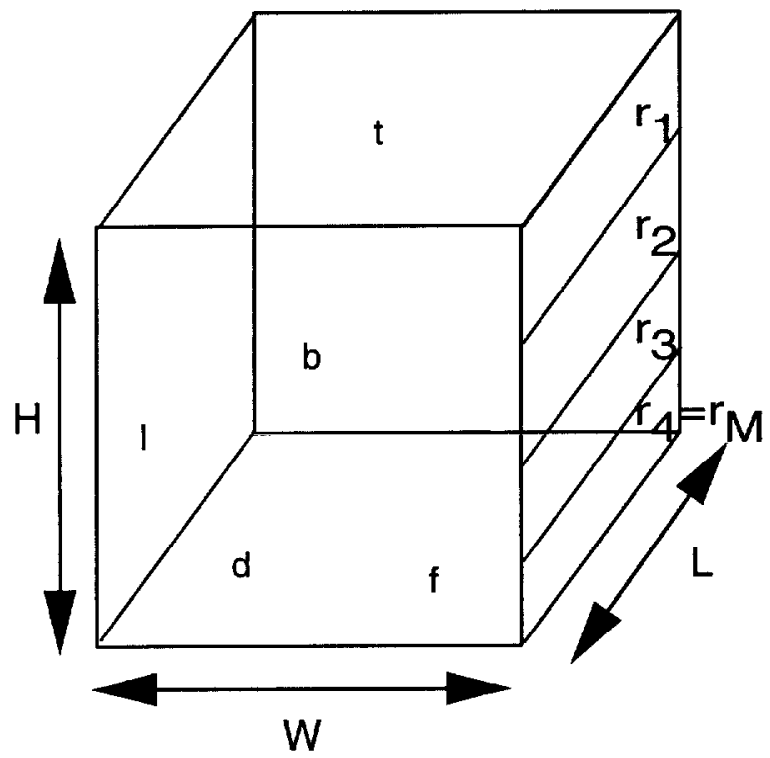

Figure 2: Setup for radiation heat transfer calculation in a room whose walls are divided into $\mathrm{M}$ horizontal strips. The faces or wall surfaces are labeled $u, f, r, b, l$ and $d$ for top, front, right, back, left and down respectively. The width, length and height of the compartment are given by $\mathrm{W}, \mathrm{L}$ and $\mathrm{H}$ respectively. The $i$ 'th strip from the top of the right face is given by $r_{i}$. Strips for other faces are indicated similarly.

are much more efficient than direct methods such as Gaussian elimination, can then be used to solve the required linear system yielding the net fluxes.

\subsection{The Net Radiation Equation}

Given a collection of $N$ wall surfaces, labeled $k=1, \cdots, N$, the net flux, $\Delta q_{k}^{\prime \prime}$, incident on the $k^{\prime}$ th wall surface is given by (see equation (17-10) in [6])

$$
\frac{\Delta q_{k}^{\prime \prime}}{\varepsilon_{k}}-\sum_{j=1}^{N} \frac{1-\varepsilon_{j}}{\varepsilon_{j}} \Delta q_{j}^{\prime \prime} F_{k-j} \tau_{j-k}=\sigma T_{k}^{4}-\sum_{j=1}^{N} \sigma T_{j}^{4} F_{k-j} \tau_{j-k}-\frac{c_{k}}{a_{k}}
$$

where $F_{k-j}$ is the fraction of radiant energy given off by surface $k$ that is intercepted by surface $j$ and $\tau_{j-k}$ is the fraction of radiant energy passing unimpeded from surface $j$ to surface $k, T_{k}$ and $\varepsilon_{k}$ are the temperature and emissivity of the $k^{\prime}$ th surface and $c_{k} / a_{k}$ is the heat flux striking surface $k$ due to fire and gas layer emission. The term $c_{k} / a_{k}$ is given by

$$
\frac{c_{k}}{a_{k}}=\sum_{\text {panels } j}\left(q_{j-k}^{\prime \prime L, g a s}+q_{j-k}^{\prime \prime U, g a s}\right)+\sum_{\text {fires } f} q_{f-k}^{\prime \prime f i r e}
$$




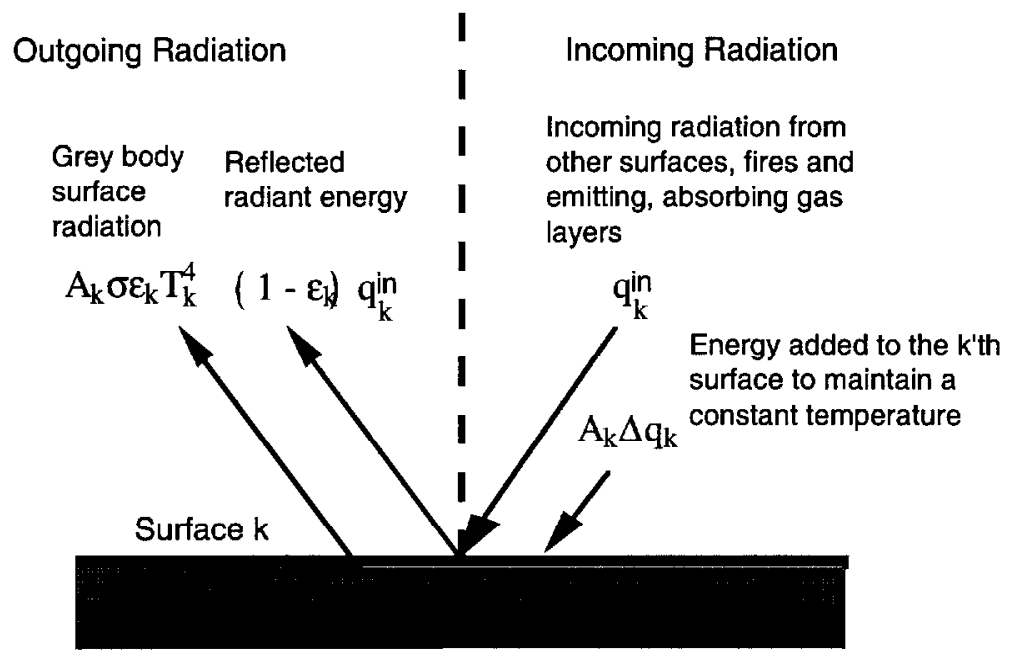

Figure 3: Input and Output Energy Distribution at the k'th Wall Surface

where $q_{f-k}^{\prime \prime f i r e}$ is given by equation (89), $q_{j-k}^{\prime \prime L, g a s}$ is given by equation (90) and $q_{j-k}^{\prime \prime U \text {,gas }}$ is given by equation (91). The radiative interaction at a single wall surface is illustrated in Figure 3 where $A_{k}, \varepsilon_{k}, T_{k}, q_{k}^{i n}$ and $\Delta q_{k}$ are the area, emissivity, temperature, incoming radiative heat flux and net radiative heat flux at the $k^{\prime}$ th wall surface respectively.

The flux calculated in (19) can be used as a boundary condition for an associated heat conduction problem in order to compute wall surface temperatures at a subsequent time step.

To make the equations more compact, define

$$
\begin{aligned}
\mathrm{A}_{j k} & = \begin{cases}1 & j=k \\
-\left(1-\varepsilon_{j}\right) F_{k-j} \tau_{j-k} & j \neq k\end{cases} \\
B_{j k} & = \begin{cases}1 & j=k \\
-F_{k-j} \tau_{j-k} & j \neq k\end{cases} \\
\mathrm{b}_{k} & =\sigma \sum_{j=1}^{N} B_{j k} T_{j}^{4}+c_{k} / a_{k} \\
x_{k} & =\Delta q_{k}^{\prime \prime} / \varepsilon_{k}
\end{aligned}
$$

then equation (19) reduces to

$$
\mathrm{A} x=\mathrm{b}
$$

which can be solved directly using the Linpack routines DGEFA and DGESL[7] or iteratively using a method such as Gauss-Seidel[8]. In order to solve for the net fluxcs, $\Delta q_{k}^{\prime \prime}$, 
a

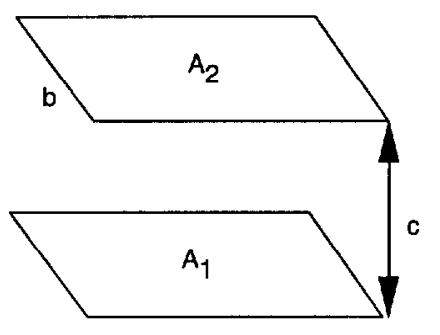

h

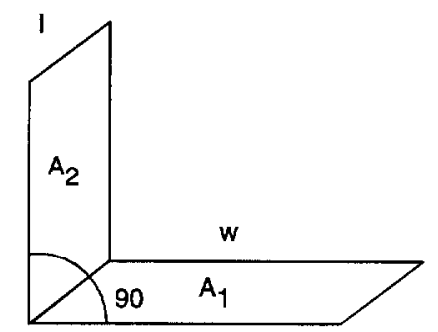

Figure 4: Configuration Factor Setup For Pairs of Parallel and Perpendicular Rectangles

in (19) or (25) configuration factors, $F_{k-j}$, transmissivities, $\tau_{j-k}$ and gas and fire radiative contributions, $c_{k}$ must first be computed. These computations are discussed in the next two sections.

\subsubsection{Configuration Factor Calculations}

In general $(4 M+2)^{2}$ configuration factors need to be computed in order to solve (19) for the net fluxes, $\Delta q^{\prime \prime}$. Due to the symmetry present in the problem illustrated in Figure 2 (horizontal strips on opposite faces are the same size) the number of configuration factors required to be computed directly in (19) can be reduced to approximately $5 M$. For $M=10$, 50 configuration factors must be computed directly rather than 1764 .

Parallel and Perpendicular Configuration Factor Formulas Consider two mutually perpendicular rectangles having a common edge of length $l$ as illustrated in Figure 4.

The dimension of the "from" rectangle is $l \times w$ and the dimension of the "to" rectangle is $l \times h$. The configuration factor, $\phi_{\text {perp }}(h, l, w)$, from rectangle 1 to 2 is then

$$
\begin{aligned}
& F_{1-2}=\phi_{\text {perp }}(h, l, w)= \\
& \quad \frac{1}{\pi W}\left\{W \operatorname{Tan}^{-1} \frac{1}{W}+H \operatorname{Tan}^{-1} \frac{1}{H}-\sqrt{H^{2}+W^{2}} \operatorname{Tan}^{-1} \frac{1}{\sqrt{H^{2}+W^{2}}}+\right. \\
& \frac{1}{4} \ln \left\{\frac{\left(1+W^{2}\right)\left(1+H^{2}\right)}{1+H^{2}+W^{2}}\left[\frac{W^{2}\left(1+H^{2}+W^{2}\right)}{\left(1+W^{2}\right)\left(H^{2}+W^{2}\right)}\right]^{W^{2}}\right. \\
& \left.\left.\quad \times\left[\frac{H^{2}\left(1+H^{2}+W^{2}\right)}{\left(1+W^{2}\right)\left(H^{2}+W^{2}\right)}\right]^{H^{2}}\right\}\right\}
\end{aligned}
$$

where $H=h / l$ and $W=w / l$. This formula is found in [6, p. 825].

We also need to compute the configuration factor between two identical, parallel, directly opposed rectangles. This situation is illustrated in Figure 4. The distance between the 
two rectangles is $c$. The length and width of these rectangles is $a$ and $b$. The configuration factor, $\phi_{\text {par }}(a, b, c)$ between these two rectangles is then

$$
\begin{aligned}
& F_{1-2}=\phi_{p a r}(a, b, c)= \\
& \quad \frac{1}{\pi X Y}\left\{\ln \sqrt{\frac{\left(1+X^{2}\right)\left(1+Y^{2}\right)}{1+X^{2}+Y^{2}}}+X \sqrt{1+Y^{2}} \operatorname{Tan}^{-1} \frac{X}{1+Y^{2}}+\right. \\
& \left.Y \sqrt{1+X^{2}} \operatorname{Tan}^{-1} \frac{Y}{1+X^{2}}\right\} .
\end{aligned}
$$

where $X=a / c$ and $Y=b / c$. This formula is symmetric in $X$ and $Y$. It can be found in [6, p. 824]. Equations (26) and (27) will be used to compute configuration factors between every pair of panels/strips.

Configuration Factors Between Wall Strips Let the faces in Figure 2 be labeled: $u, f$, $r, b, l$ and $d$ for up, front, right, back, left and down respectively. Assume that the vertical faces labled: front, right, back and left are divided into $M$ horizontal strips of equal height. Strips on faces $f$ and $b$ each have equal area. Similarly, strips on faces $r$ and $l$ each have equal area.

The notation, $F_{m_{j}-n_{k}}$ denotes the configuration factor from the $j^{\prime} t h$ strip on face $m$ to the $k^{\prime}$ th strip on face $n$ where $m$ and $n$ can be any of $u, f, r, b, l$ or $d$ and $1 \leq j, k \leq M$. Note that the top and bottom faces are not divided into strips.

To compute the $(4 M+2)^{2}$ configuration factors, first compute the parallel configuration factor between the up and down surface:

$$
F_{u-d}=\phi_{p a r}(W, L, H)
$$

Next, using $\Delta H=H / M$ and $i=1, \cdots, M$ compute the configuration factors between surface $u$ to each $f_{i}$; surface $u$ to each $r_{i}$; surface $f_{1}$ to each $r_{i}$; surface $f_{1}$ to each $b_{i}$; and surface $r_{1}$ to each $l_{i}$ using:

$$
\begin{aligned}
& F_{u-f_{i}}=\phi_{\text {perp }}(i \Delta H, W, L)-\phi_{\text {perp }}((i-1) \Delta H, W, L) \\
& F_{u-r_{i}}= \phi_{\text {perp }}(i \Delta H, L, W)-\phi_{\text {perp }}((i-1) \Delta H, L, W) \\
& F_{f_{1}-r_{i}}= \begin{cases}\phi_{\text {perp }}(L, \Delta H, W) & i=1 \\
\left(i \phi_{\text {perp }}(L, i \Delta H, W)-\right. & (i-1) \phi_{\text {perp }}(L,(i-1) \Delta H, W)- \\
\left.\phi_{\text {perp }}(L, \Delta H, W)\right) / 2 & i \neq 1\end{cases} \\
& F_{f_{1}-b_{i}}= \begin{cases}\phi_{\text {par }}(\Delta H, W, L) \\
\left(i \phi_{\text {par }}(i \Delta H, W, L)-(i-1) \phi_{\text {par }}((i-1) \Delta H, W, L)-\right. & i=1 \\
\left.\phi_{\text {par }}(\Delta H, W, L)\right) / 2 & i \neq 1\end{cases}
\end{aligned}
$$




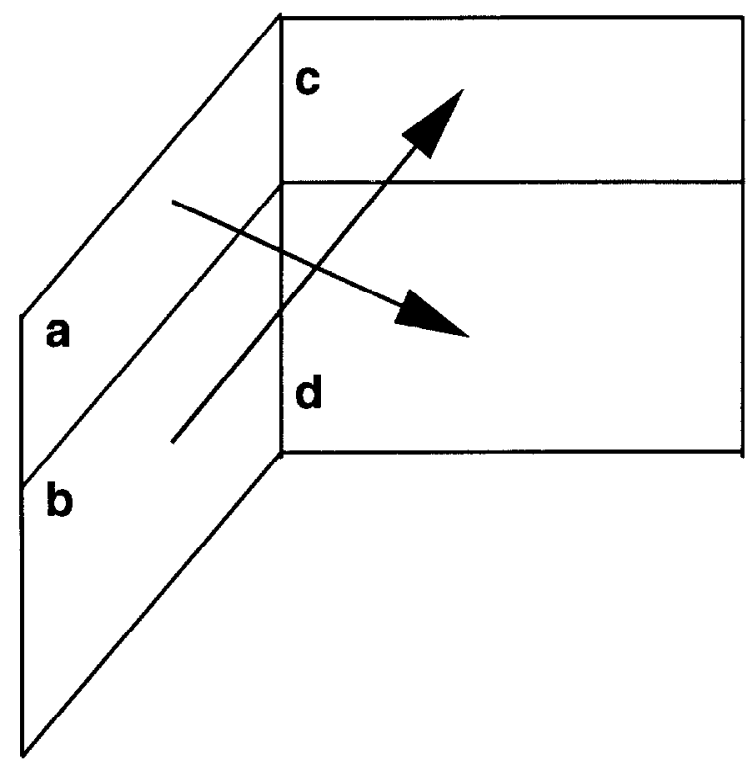

Figure 5: Diagram illustrating the configuration factor identity, $A_{a b} F_{a-d}=A_{c d} F_{b-d}$, where $A_{a b}$ denotes the combined areas of surfaces a and $\mathrm{b}$ and $A_{c d}$ is defined analogously.

$$
F_{r_{1}-l_{i}}= \begin{cases}\phi_{p a r}(\Delta H, L, W) & i=1 \\ \left(i \phi_{\text {par }}(i \Delta H, L, W)-(i-1) \phi_{\text {par }}((i-1) \Delta H, L, W)-\right. & \\ \left.\phi_{\text {par }}(\Delta H, L, W)\right) / 2 & i \neq 1\end{cases}
$$

The above configuration factors can be derived using the identity illustrated in Figure 5 and

$$
\begin{aligned}
A_{a b} F_{a b-c d} & =A_{a} F_{a-c d}+A_{b} F_{b-c d} \\
& =A_{a}\left(F_{a-c}+F_{a-d}\right)+A_{b}\left(F_{b-c}+F_{b-d}\right) \\
& =A_{a} F_{a-c}+2 A_{a} F_{a-d}+A_{b} F_{b-d} \\
F_{a-d} & =\frac{A_{a b} F_{a b-c d}-A_{a} F_{a-c}-A_{b} F_{b-d}}{2} .
\end{aligned}
$$

For example, to derive equation (31), let $A_{a}=W \Delta H, A_{b}=W(i-1) \Delta H, A_{c}=L \Delta H$ and $A_{d}=(i-1) \Delta H L$.

Finally use these $5 M+1$ configuration factors to compute all $(4 M+2)^{2}$ configuration factors in the following way.

\section{Factors from surface $u$}

$$
\begin{aligned}
F_{u-b_{i}} & =F_{u-f_{i}} \\
F_{u-l_{i}} & =F_{u-r_{i}}
\end{aligned}
$$


Factors $F_{u-d}, F_{u-f_{i}}$ and $F_{u-r_{i}}$ are defined using equations (28), (29) and (30) respectively.

Factors from surface $f_{i}$

$$
\begin{aligned}
F_{f_{i}-r_{j}} & =F_{f_{1}-r_{1+|j-i|}} \\
F_{f_{i}-b_{j}} & =F_{f_{1}-b_{1+|j-i|}} \\
F_{f_{i}-l_{j}} & =F_{f_{i}-r_{j}}=F_{f_{1}-r_{1+|j-i|}} \\
F_{f_{i}-d} & =F_{f_{i}-u}=\frac{A_{u}}{A_{f_{i}}} F_{u-f_{i}}
\end{aligned}
$$

where $A_{u}=L W, A_{f_{i}}=W \Delta H$ and $A_{u} / A_{f_{i}}=L / \Delta H$.

\section{Factors from surface $r_{i}$}

$$
\begin{aligned}
F_{r_{i}-b_{j}} & =F_{r_{i}-f_{j}}=\frac{A_{f_{j}}}{A_{r_{i}}} F_{f_{j}-r_{i}} \\
F_{r_{i}-l_{j}} & =F_{r_{1}-l_{1+|j-i|}} \\
F_{r_{i}-d} & =F_{r_{i}-u}-\frac{A_{u}}{A_{r_{i}}} F_{u-r_{i}}
\end{aligned}
$$

where $A_{r_{i}}=L \Delta H, A_{f_{j}} / A_{r_{i}}=W / L$ and $A_{u} / A_{r_{i}}=W / \Delta H$.

\section{Factors from surface $b_{i}$}

$$
\begin{aligned}
& F_{b_{i}-r_{j}}=F_{b_{i}-l_{j}}=F_{f_{i}-r_{j}} \\
& F_{b_{i}-d}=F_{b_{i}-u}=F_{f_{i}-u}=\frac{A_{u}}{A_{f_{i}}} F_{u-f_{i}} \\
& F_{b_{i}-f_{j}}=F_{f_{j}-b_{i}}=F_{f_{1}-b_{1+|j-i|}}
\end{aligned}
$$

Factors from surface $l_{i}$ By symmetry, a configuration factor from a left face $l_{i}$ to any face $x_{y}$ is identical to the configuration factor from the corresponding right face $r_{i}$ or equivalently

$$
F_{l_{i}-x_{y}}=F_{r_{i}-x_{y}}
$$

Factors from surface $d$ By symmetry, a configuration factor from face $d$ to any face $x_{y}$ is identical to the configuration factor from face $u$ or equivalently

$$
F_{d-x_{y}}=F_{u-x_{y}}
$$




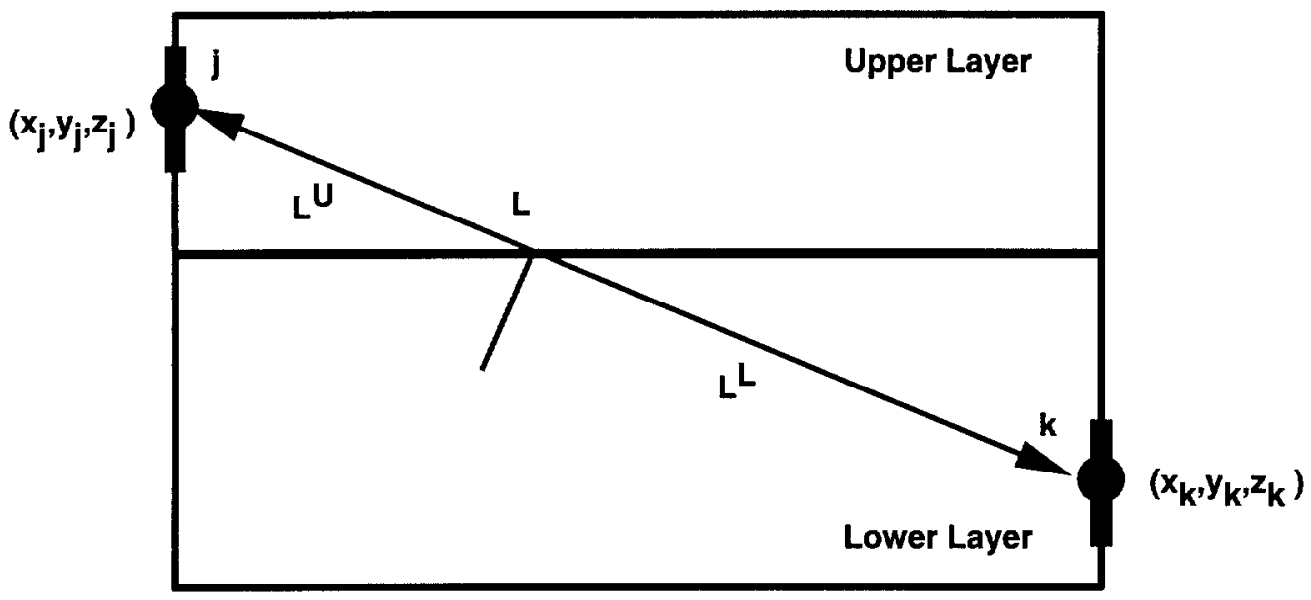

Figure 6: Cross-sectional view of a compartment with wall surfaces $j$ and $k$. The path between $j$ and $k$ has length $L$. The portion of the path in the lower layer has length $L^{L}$ and the portion in the upper layer has length $L^{U}$.

\subsubsection{Transmissivities}

The transmissivity, $\tau_{j-k}$, in equation (19) is given by $\tau_{j-k}=\exp \left(-a_{U} L^{U}\right) \exp \left(-a_{L} L^{L}\right)$ where $a_{L}$ and $a_{U}$ are the absorptivities of the lower and upper layers respectively and $L^{L}$ and $L^{U}$ are the path lengths from $j$ to $k$ in the lower layer and upper layer respectively as illustrated in Figure 6.

The quantities, $L^{L}$ and $L^{U}$, can be computed using proportionalities. First note that the distance $L$ from panels $j$ to $k$ is given by

$$
L=\sqrt{\left(x_{j}-x_{k}\right)^{2}+\left(y_{j}-y_{k}\right)^{2}+\left(z_{j}-z_{k}\right)^{2}}
$$

where $\left(x_{j}, y_{j}, z_{j}\right)$ and $\left(x_{k}, y_{k}, z_{k}\right)$ are the center coordinates of panels $j$ and $k$. The distances, $L^{L}$ and $L^{U}$ are given by

$$
\begin{aligned}
L^{U} / L & = \begin{cases}1 & z_{j} \geq y_{\text {lay }} \text { and } z_{k} \geq y_{\text {lay }} \\
\left(\max \left(z_{j}, z_{k}\right)-y_{\text {lay }}\right) /\left|z_{j}-z_{k}\right| & z_{j} \geq y_{\text {lay }} \geq z_{k} \text { or } z_{k} \geq y_{\text {lay }} \geq z_{j} \\
0 & y_{\text {lay }} \geq z_{j} \text { and } y_{\text {lay }} \geq z_{k}\end{cases} \\
L^{L} & =L-L^{U}
\end{aligned}
$$

The fraction of radiant energy, $\tau_{j_{m}-k_{n}}^{\text {wall }}$, transmitted through the upper layer between the $m^{\prime}$ th strip of face $j$ and the $n^{\prime}$ th strip of face $k$ is given by

$$
\tau_{u-f_{n}}^{\text {wall,U }}=\exp \left(-a_{U} L_{u-f_{n}}^{U}\right)
$$




$$
\begin{aligned}
& \tau_{f_{m}-r_{n}}^{\text {wall } U}= \begin{cases}\tau_{f_{1}-r_{1+|m-n|}}^{\text {wall },} & z_{m}>y_{l a y} \text { and } z_{n}>y_{l a y} \\
1 & z_{m}<y_{l a y} \text { and } z_{n}<y_{l a y} \\
\exp \left(-a_{U} L_{f_{m}-r_{n}}^{U}\right) & \text { otherwise }\end{cases} \\
& \tau_{f_{m}-b_{n}}^{\text {wall } U}= \begin{cases}\tau_{f_{1}-b_{1+|m-n|}}^{\text {wall } U} & z_{m}>y_{\text {lay }} \text { and } z_{n}>y_{\text {lay }} \\
1 & z_{m}<y_{\text {lay }} \text { and } z_{n}<y_{\text {lay }} \\
\exp \left(-a_{U} L_{f_{m}-b_{n}}^{U}\right) & \text { otherwise }\end{cases} \\
& \tau_{f_{m}-l_{n}}^{\text {wall } U}=\tau_{f_{m}-r_{n}}^{\text {wall } U} \\
& \tau_{f_{m}-d}^{\text {wall,U}}=\exp \left(-a_{U} L_{f_{m}-d}^{U}\right) \\
& \tau_{r_{m}-b_{n}}^{\text {wall } U}=\tau_{f_{m}-r_{n}}^{\text {wall } U} \\
& \tau_{r_{m}-l_{n}}^{\text {wall,U }}= \begin{cases}\tau_{r_{1}-l_{1+|m-n|}}^{\text {wall } U} & z_{m}>y_{\text {lay }} \text { and } z_{n}>y_{\text {lay }} \\
1 & z_{m}<y_{\text {lay }} \text { and } z_{n}<y_{\text {lay }} \\
\exp \left(-a_{U} L_{r_{m}-l_{n}}^{U}\right) & \text { otherwise }\end{cases} \\
& \tau_{r_{m}-d}^{w a l l, U}=\exp \left(-a_{U} L_{r_{m}-d}^{U}\right) \\
& \tau_{b_{m}-l_{n}}^{\text {wall,U }}=\tau_{f_{m}-r_{n}}^{\text {wall, }} \\
& \tau_{b_{m}-d}^{\text {wall }, U}=\tau_{f_{m}-d}^{\text {wall }, U}
\end{aligned}
$$

Also note that by symmetry

$$
\tau_{j_{m}-k_{n}}^{\text {wall,U}}=\tau_{k_{n}-j_{m}}^{\text {wall },}
$$

where faces $j$ and $k$ can be any of $u, f, r, b, l, d$.

Similarly, the fraction of radiant energy, $\tau_{j_{m}-k_{n}}^{w a l l, L}$, transmitted through the lower layer between the $m$ 'th strip of face $j$ and the $n$ 'th strip of face $k$ is given by

$$
\begin{aligned}
\tau_{u-f_{n}}^{\text {wall }, L} & =\exp \left(-a_{L} L_{u-f_{n}}^{L}\right) \\
\tau_{f_{m}-r_{n}}^{\text {wall } L} & = \begin{cases}\tau_{f_{1}-r_{1+|m-n|}^{\text {wall }, L}} & z_{m}<y_{\text {lay }} \text { and } z_{n}<y_{\text {lay }} \\
1 & z_{m}>y_{\text {lay }} \text { and } z_{n}>y_{\text {lay }} \\
\exp \left(-a_{L} L_{f_{m}-r_{n}}^{L}\right) & \text { otherwise }\end{cases} \\
\tau_{f_{m}-b_{n}}^{\text {wall }, L} & = \begin{cases}\tau_{f_{1}-b_{1+|m-n|} \text { wall }, L} & z_{m}<y_{\text {lay }} \text { and } z_{n}<y_{\text {lay }} \\
1 & z_{m}>y_{\text {lay }} \text { and } z_{n}>y_{\text {lay }} \\
\exp \left(-a_{L} L_{f_{m}-b_{n}}^{L}\right) & \text { otherwise }\end{cases} \\
\tau_{f_{m}-l_{n}}^{\text {wall }, L} & =\tau_{f_{m}-r_{n}}^{\text {wall } L} \\
\tau_{f_{m}-d}^{\text {wall }, L} & =\exp \left(-a_{L} L_{f_{m}-d}^{L}\right)
\end{aligned}
$$




$$
\begin{aligned}
\tau_{r_{m}-b_{n}}^{\text {wall } L} & =\tau_{f_{m}-r_{n}}^{\text {wall } L} \\
\tau_{r_{m}-l_{n}}^{\text {wall }, L} & = \begin{cases}\tau_{r_{1}-l_{1+|m-n|}}^{\text {wall }, L} & z_{m}<y_{\text {lay }} \text { and } z_{n}<y_{\text {lay }} \\
1 & z_{m}>y_{\text {lay }} \text { and } z_{n}>y_{\text {lay }} \\
\exp \left(-a_{L} L_{r_{m}-l_{n}}^{L}\right) & \text { otherwise }\end{cases} \\
\tau_{r_{m}-d}^{\text {wall }, L} & =\exp \left(-a_{L} L_{r_{m}-d}^{L}\right) \\
\tau_{b_{m}-l_{n}}^{\text {wall }, L} & =\tau_{f_{m}, L_{n}}^{\text {wall },} \\
\tau_{b_{m}-d}^{\text {wall }, L} & =\tau_{f_{m}-d}^{\text {wall,L }}
\end{aligned}
$$

Again note that by symmetry

$$
\tau_{j_{m}-k_{n}}^{\text {wall,L}}=\tau_{k_{n}-j_{m}}^{\text {wall, }}
$$

where faces $j$ and $k$ can be any of $u, f, r, b, l, d$.

The fractions of radiant energy, $\tau_{f-k_{n}}^{f i r e, L}$ and $\tau_{f-k_{n}}^{\text {fire } U}$, transmitted through the lower and upper layers between the $f^{\prime}$ th fire and the $n^{\prime}$ th strip of face $k$ are given by

$$
\begin{aligned}
\tau_{f-k_{n}}^{\text {fire } L} & = \begin{cases}1 & z_{f}>y_{\text {lay }} \text { and } z_{k}>y_{\text {lay }} \\
\exp \left(-a_{L} L^{L}\right) & \text { otherwise }\end{cases} \\
\tau_{f-k_{n}}^{\text {fire } U} & =\left\{\begin{array}{ll}
1 & z_{f}<y_{\text {lay }} \text { and } z_{k}<y_{\text {lay }} \\
\exp \left(-a_{U} L^{U}\right) & \text { otherwise }
\end{array} .\right.
\end{aligned}
$$

\subsubsection{Solid angles}

A solid angle of an object with respect to a point $x_{f}$ is the area intercepted on a unit sphere by a conical angle originating at the sphere center. In our application a solid angle is used to determine the fraction of a radiating point source fire that strikes a surface.

In this section we wish to find the solid angle between a point $x_{f}$ and a triangle formed by arbitrary points $\hat{x}_{1}, \hat{x}_{2}$ and $\hat{x}_{3}$. The solid angle of any object can then be found by triangulating the object.

First we subtract off the point $x_{f}$ from each $\hat{x}_{i}$ to obtain

$$
x_{i}=\hat{x}_{i}-x_{f}
$$

Let $r$ be the length of the shortest vector $x_{i}$

$$
r=\min \left(\left\|x_{1}\right\|,\left\|x_{2}\right\|,\left\|x_{3}\right\|\right)
$$

where $\|\cdot\|$ has the usual meaning $\|a\|=\sqrt{a_{x}^{2}+a_{y}^{2}+a_{z}^{2}}$ for the vector $a=\left(a_{x}, a_{y}, a_{z}\right)$. Let $p_{1}, p_{2}, p_{3}$ be points on a sphere of radius $r$ co-linear with $x_{i}$ and the zero vector. The point 


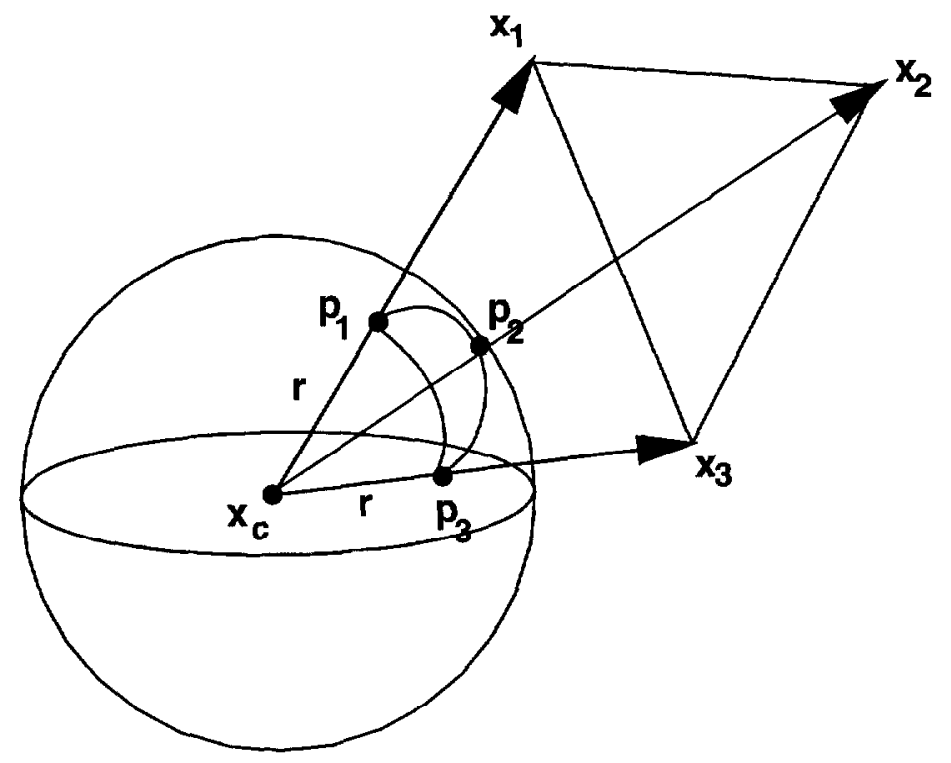

Figure 7: Solid Angle Setup For an Arbitrarily Oriented Triangle

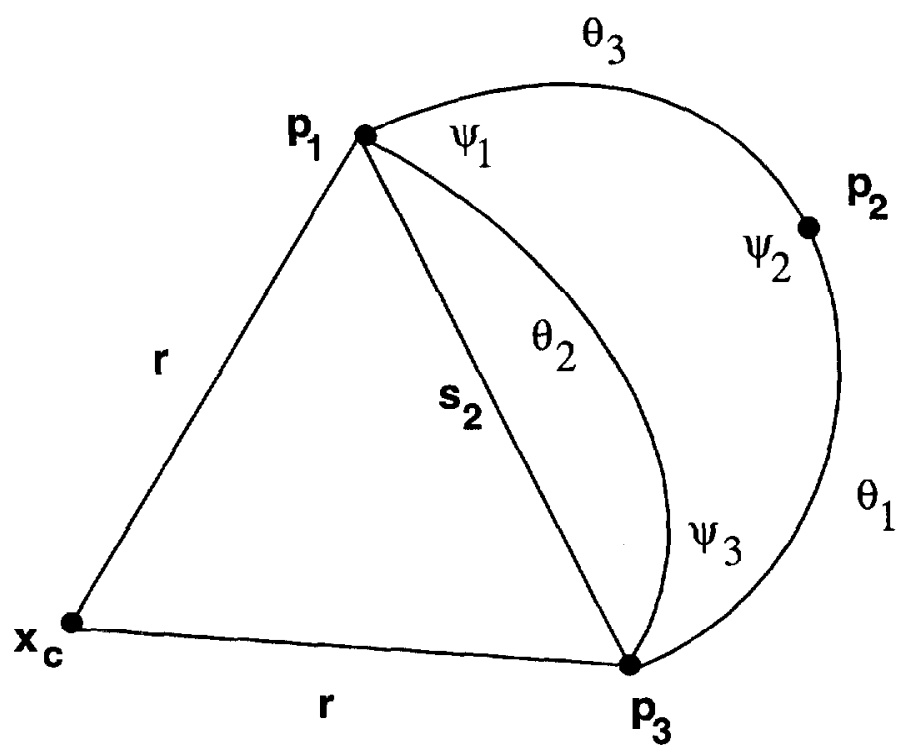

Figure 8: Solid Angle Setup For an Arbitrarily Oriented Triangle 
$p_{i}$ is then given by

$$
p_{i}=\frac{x_{i}}{\left\|x_{i}\right\|} r
$$

These points then form a "spherical" triangle. The area of this triangle normalized by $r^{2}$ is then the solid angle which we are seeking.

Let $s_{i}$ be the distances between between the points $p_{1}, p_{2}$ and $p_{3}$ given by

$$
\begin{aligned}
& s_{1}=\left\|p_{2}-p_{3}\right\| \\
& s_{2}=\left\|p_{1}-p_{3}\right\| \\
& s_{3}=\left\|p_{1}-p_{2}\right\|
\end{aligned}
$$

The angle corresponding to $s_{i}$ is denoted $\theta_{i}$. The cosine and sine of this angle can be found using $[9$, p. 238] and is given by

$$
\begin{aligned}
\cos \theta_{i} & =1-\frac{s_{i}^{2}}{2 r^{2}} \\
\sin \theta_{i} & =\frac{s_{i}}{r} \sqrt{1-\frac{s_{i}^{2}}{4 r^{2}}}
\end{aligned}
$$

The angle opposite of side with length $s_{i}$ is denoted $\psi_{i}$ and is given by

$$
\psi_{i}=\arccos \left(\frac{\cos \theta_{i}-\Pi_{j=1}^{3} \cos \theta_{j}}{\prod_{j=1}^{3} \sin \theta_{j}}\right)
$$

Finally the solid angle of a triangle with points, $x_{1}, x_{2}$ and $x_{3}$ with respect to $x_{f}$ is given by

$$
\psi\left(x_{f}, x_{1}, x_{2}, x_{3}\right)=\psi_{1}+\psi_{2}+\psi_{3}-\pi
$$

The solid angle of a parallelogram $k$ with base point $x_{k_{1}}$ and direction vectors $d_{k_{1}}$ and $d_{k_{2}}$ with respect to the base point of a fire $x_{f}$ can be found by dividing the parallelogram into two tri-angles and is given by

$$
\begin{aligned}
\omega_{f-k}= & \psi\left(x_{f}, x_{k_{1}}, x_{k_{1}}+d_{k_{1}}, x_{k_{1}}+d_{k_{2}}\right)+ \\
& \psi\left(x_{f}, x_{k_{1}}+d_{k_{1}}, x_{k_{1}}+d_{k_{1}}+d_{k_{2}}, x_{k_{1}}+d_{k_{2}}\right)
\end{aligned}
$$

\subsubsection{Computing the source term, $c$}

Contributions from Fires If $q_{f}$ is the energy release rate of a fire and $\chi$ is the fraction of the fire that radiates then the flux striking the $k$ 'th surface due to fire $f$ is

$$
q_{f-k}^{\prime \prime f i r e}=\chi \frac{\omega_{f-k}}{4 \pi a_{k}} q_{f} \tau_{f-k}^{\text {fire } L} \tau_{f-k}^{\text {fire } U}
$$




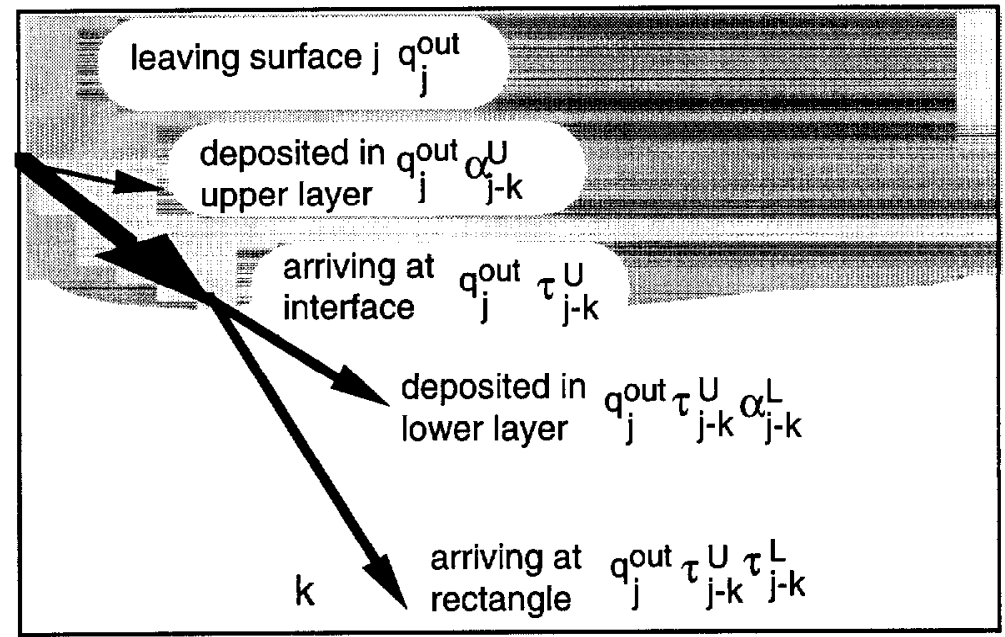

Figure 9: Schematic illustrating energy deposited into the upper layer, the lower layer and arriving at the $k^{\prime} t h$ wall surface due to the $j^{\prime} t h$ wall surface.

Contributions From Gas Layers The flux striking a wall panel $k$ due to the lower and upper gas layer along a path from panel $j$ to $k$ is given by

$$
\begin{aligned}
& q_{j-k}^{\prime \prime L, g a s}= \begin{cases}0 & z_{j}>y_{\text {lay }} \text { and } z_{k}>y_{\text {lay }} \\
\sigma F_{k-j} \alpha_{j-k}^{L} T_{L}^{4} & z_{k}<y_{\text {lay }} \\
\sigma F_{k-j} \alpha_{j-k}^{L} T_{L}^{4} \tau_{j-k}^{L} & z_{j}<y_{\text {lay }} \text { and } z_{k}>y_{\text {lay }}\end{cases} \\
& q_{j-k}^{\prime \prime \prime}{ }_{j, \text { gas }}= \begin{cases}0 & z_{j}<y_{\text {lay }} \text { and } z_{k}<y_{\text {lay }} \\
\sigma F_{k-j} \alpha_{\dot{-k}}^{U} T_{U}^{4} & z_{k}>y_{\text {lay }} \\
\sigma F_{k-j} \alpha_{j-k}^{U} T_{U}^{4} \tau_{j-k}^{U} & z_{j}>y_{\text {lay }} \text { and } z_{k}<y_{\text {lay }}\end{cases}
\end{aligned}
$$

\subsubsection{Radiant Heat Absorbed by Gas Layers}

Contributions from Walls The total heat (including re-radiation), $q_{j-k}^{\text {out }}$, emitted by panel $j$ along a direction towards the panel $k$ is illustrated in Figure 9 and given by

$$
q_{j-k}^{\text {out }}=A_{j} F_{j-k}\left(\sigma T_{j}^{4}-\frac{1-\varepsilon_{j}}{\varepsilon_{j}} \Delta q_{j}^{\prime \prime}\right)
$$


The radiant heat absorbed by the upper and lower gas layers due to this emission is given by (see Tables 2 and 3 in [3])

$$
\begin{aligned}
q_{j-k}^{\text {wall }, L} & = \begin{cases}q_{j-k}^{\text {out }} \alpha_{j-k}^{\text {wall }, L} & z_{j}<y_{\text {lay }} \\
q_{j-k}^{\text {out }} \alpha_{j-k}^{\text {wall }, L} \tau_{j-k}^{\text {wall }, L} & z_{j}>y_{\text {lay }} \text { and } z_{k}<y_{\text {lay }} \\
0 & z_{j}>y_{\text {lay }} \text { and } z_{k}>y_{\text {lay }}\end{cases} \\
q_{j-k}^{\text {wall }, U} & = \begin{cases}q_{j-k}^{\text {out }} \alpha_{j-k}^{\text {wall }, U} & z_{j}>y_{\text {lay }} \\
q_{j-k}^{\text {out }} \alpha_{j-k}^{\text {wall }, U} \tau_{j-k}^{\text {wall }, U} & z_{j}<y_{\text {lay }} \text { and } z_{k}>y_{\text {lay }} \\
0 & z_{j}>y_{\text {lay }} \text { and } z_{k}>y_{\text {lay }}\end{cases}
\end{aligned}
$$

Contributions from Gas Layers Let $Q_{j-k}^{g a s, L}$ and $Q_{j-k}^{g a s, U}$ be given by

$$
\begin{aligned}
& Q_{j-k}^{\text {gas }, L}=A_{j} F_{j-k} \alpha_{j-k}^{\text {wall }, L} \sigma T_{L}^{4} \\
& Q_{j-k}^{\text {gas, }, J}=A_{j} F_{j-k} \alpha_{j-k}^{\text {wall }, U} \sigma T_{U}^{4}
\end{aligned}
$$

Then the radiant heat absorbed by the upper and lower gas layers due to gas layer emission is

$$
\begin{aligned}
q_{j-k}^{\text {gas }, L} & = \begin{cases}-Q_{j-k}^{\text {gas }, L} & z_{j}>y_{\text {lay }} \\
Q_{j-k}^{\text {gas,U }} \alpha_{j-k}^{\text {wall }, L}-Q_{j-k}^{\text {gas }, L} & z_{j}<y_{\text {lay }} \text { and } z_{k}>y_{\text {lay }} \\
0 & z_{j}<y_{\text {lay }} \text { and } z_{k}<y_{\text {lay }}\end{cases} \\
q_{j-k}^{\text {gas }, U} & = \begin{cases}-Q_{j-k}^{\text {gas }, U} & z_{j}<y_{\text {lay }} \\
Q_{j-k}^{\text {gas, },} \alpha_{j-k}^{\text {wall }, U}-Q_{j-k}^{\text {gas }, U} & z_{j}>y_{\text {lay }} \text { and } z_{k}<y_{\text {lay }} \\
0 & z_{j}>y_{\text {lay }} \text { and } z_{k}>y_{\text {lay }}\end{cases}
\end{aligned}
$$

Contributions from Fires Let $q_{f-k}^{\prime \prime f i r e}=\chi \frac{\omega_{f-k}}{4 \pi a_{k}} q_{f}$. Then the radiant heat absorbed by the upper and lower gas layers due to fire emission is illustrated by Figure 10 and given by

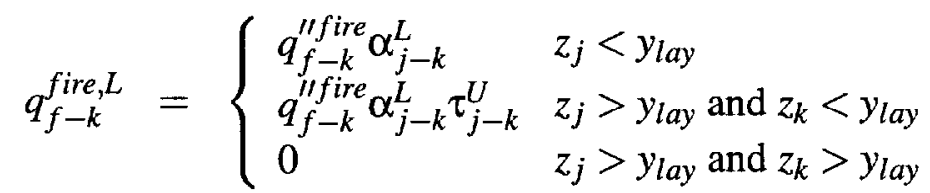

$$
\begin{aligned}
& q_{f-k}^{\text {fire } U}= \begin{cases}q_{f-k}^{\prime \prime f i r e} \alpha_{j-k}^{U} & z_{j}>y_{\text {lay }} \\
q_{f-k}^{\prime \prime \text { fire }} \alpha_{j-k}^{U} \tau_{j-k}^{L} & z_{j}<y_{\text {lay }} \text { and } z_{k}>y_{\text {lay }} \\
0 & z_{j}<y_{\text {lay }} \text { and } z_{k}<y_{\text {lay }}\end{cases}
\end{aligned}
$$




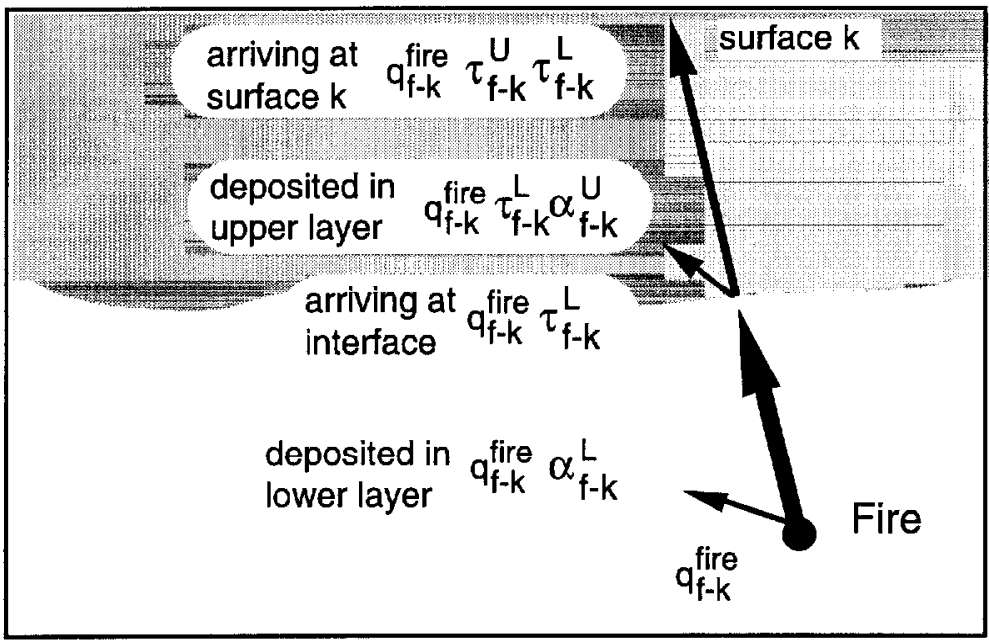

Figure 10: Schematic illustrating energy deposited into the lower layer, into the upper layer, and arriving at the $k^{\prime} t h$ wall surface due to the $f^{\prime} t h$ fire.

\subsection{Solution Properties}

The number of iterations required to solve the linear system of equations defined by (19) can be estimated using the gas layer absorptivities and the wall emissivities. The matrix form of (19) can be written as

$$
\mathrm{A} x=b
$$

where the $j k^{\prime}$ th component of $\mathrm{A}$ is given by

$$
\mathrm{A}_{j k}=\delta_{j k}-\left(1-\varepsilon_{j}\right) F_{k-j} \tau_{j-k}
$$

and $\delta$ is the kroneker delta function, the $k^{\prime}$ th component of $x$ is given by $b_{k}=\Delta q_{k}^{\prime \prime} / \varepsilon_{k}$ and the $k^{\prime}$ th component of $b$ is given by

$$
b_{k}=\delta_{j k} \sigma T_{k}^{4}-\sigma T_{j}^{4} F_{k-j} \tau_{j-k}-c_{k} / a_{k} .
$$

The iteration

$$
\begin{aligned}
x_{0} & =b, \\
x_{n+1} & =b-(\mathrm{I}-\mathrm{A}) x_{n},
\end{aligned}
$$

converges to $\mathrm{A}^{-1} b$, the solution of both (19) and (101). 
Expanding the iteration (105) for several iterations, we find that

$$
\begin{aligned}
x_{0} & =b \\
x_{1} & =b-(\mathrm{I}-\mathrm{A}) b \\
x_{2} & =b-(\mathrm{I}-\mathrm{A}) b-(\mathrm{I}-\mathrm{A})^{2} b \\
& \vdots \\
x_{n} & =\left[\sum_{i=0}^{n}(\mathrm{I}-\mathrm{A})^{i}\right] b \\
& =\mathrm{A}^{-1}\left(\mathrm{I}-(\mathrm{I}-\mathrm{A})^{n+1}\right) b .
\end{aligned}
$$

The solution of $(101)$ converges (to $\mathrm{A}^{-1} b$ ) if $\|\mathrm{I}-\mathrm{A}\|_{\infty}<1$. Let $\rho=\max \tau_{j-k}\left(1-\varepsilon_{j}\right)$. Then

$$
\begin{aligned}
\|\mathrm{I}-\mathrm{A}\|_{\infty} & =\max \sum_{j=1}^{N} F_{k-j} \tau_{j-k} \varepsilon_{j} \\
\leq \rho & \\
\left\|x_{n}-x\right\|_{\infty} & =\left\|\mathrm{A}^{-1}\left(\mathrm{I}-(I-\mathrm{A})^{n+1}\right) \mathrm{b}-\mathrm{A}^{-1} \mathrm{~b}\right\|_{\infty} \\
& =\left\|\mathrm{A}^{-1}(\mathrm{I}-\mathrm{A})^{n+1} \mathrm{~b}\right\|_{\infty} \\
& \leq\left\|\mathrm{A}^{-1}\right\|\left\|_{\infty} \rho^{n+1}\right\| \mathrm{b} \|_{\infty}
\end{aligned}
$$

Suppose that each wall surface has an emissivity of $\varepsilon=0.9$ Then $\rho \leq 1-0.9=0.10$. If the error in the initial guess is 1 , i.e. $\|b\|_{\infty}=1$ then the error in the net radiation flux calculated iteratively using equations (19) and (105) after 5 iterations is smaller than $\rho^{5}=$ $10^{-5}$.

Mathematically, the number of iterations required for convergence is related to the matrix norm, $\|\mathrm{I}-\hat{\mathrm{A}}\|_{\infty}$, where the $j k^{\prime}$ th component of $\hat{\mathrm{A}}$ is given by

$$
\hat{A}_{j k}=\delta_{j k}-\left(1-\varepsilon_{j}\right) F_{k-j} \tau_{j-k}
$$

and $\delta$ is the kroneker delta function.

\section{RADSTRIP0 - A simplified approach to computing ra- diation exchange}

The discussion in previous sections assumes that wall strip surface temperatures are independent. The approach described here uses the simpler RAD4 radiation algorithm in 
conjunction with wall strip heat conduction by combining several wall strips into one for the purposes of a radiation exchange algorithm. The algorithms used to implement RADSTRIP may be simplified considerably (at the expense of accuracy) by grouping several wall strips into one surface and then choosing appropriate temperatures to represent the overall surface. For example, consider the strips on the right face in Figure 2. The total energy radiated by these strips is found by summing the contributions from each strip to obtain

$$
E_{t o t}=\sigma \sum_{k} a_{k} \varepsilon_{k} T_{k}^{4}
$$

where $\Sigma_{k}$ sums over all surfaces of interest. Assume that there is no mutual interaction between the strips. An average temperature may then be obtained by estimating an average emissivity and requiring the average temperature to satisfy the relation

$$
E_{\text {tot }}=\sigma \varepsilon_{\mathrm{avg}} T_{\mathrm{avg}}^{4} \sum_{k} a_{k}
$$

If $\varepsilon_{\text {avg }}$ is the arithmetic average based on strip emissivities where areas are used as weights i.e.

$$
\varepsilon_{\mathrm{avg}}=\frac{\sum_{k} a_{k} \varepsilon_{k}}{\sum_{k} a_{k}}
$$

then $T_{\text {avg }}$ may be approximated using

$$
T_{\text {avg }}=\sqrt[4]{\frac{\sum_{k} a_{k} \varepsilon_{k} T_{k}^{4}}{\sum_{k} a_{k} \varepsilon_{k}}}
$$

Radiation exchange between all wall surfaces then estimated by 1 ) computing $T_{\text {avg }}$ values for strips forming upper and lower walls 2) passing these temperatures to RAD4 (along with ceiling and floor temperatures), 3) using fluxes computed by RAD4 for upper and lower walls to estimate fluxes for each wall strip.

\section{Summary}

Inter-compartment heat transport theory and algorithms based on the work of Moss[2] were presented. New radiative heat transfer algorithms were designed in order to provide boundary conditions consistent with the conduction algorithms. Convection algorithms remain substantially the same as are now present in CFAST 3.1.3. Procedures are outlined in the appendices on how these algorithms may be implemented in the zone fire model CFAST. 


\section{References}

[1] Richard D. Peacock, Glenn P. Forney, Paul Reneke, Rebecca Portier, and Walter W. Jones. CFAST, the consolidated model of fire growth and smoke transport. Technical Note 1299, National Institute of Standards and Technology, 1993.

[2] William F. Moss. Computational heat transfer for zone fire modeling - summary report for nist grant no. 60nanb2d1281 covering the period june 1, 1994 to march 31, 1996. Government contract report, National Institute of Standards and Technology, 1993.

[3] Glenn P. Forney. Computing radiative heat transfer occurring in a zone fire model. Internal Report 4709, National Institute of Standards and Technology, 1991.

[4] Glenn P. Forney. Computing radiative heat transfer occurring in a zone fire model. Fire Science and Technology, 14:31-47, 1994.

[5] L. Lapidus and G. F. Pinder. Numerical Solution of Partial Differential Equations in Science and Engineering. Wiley, New York, 1982.

[6] Robert Siegel and John R. Howell. Thermal Radiation Heat Transfer. Hemisphere Publishing Corporation, New York, second edition, 1981.

[7] J. J. Dongarra, C. B. Moler, J. R. Bunch, and G. W. Stewart. LINPACK User's Guide. Society for Industrial and Applied Mathematics, Philadelphia, 1979.

[8] Gene H. Golub and James M. Ortega. Scientific Computing and Differential Equations, An Introduction to Numerical Methods. Academic Press, New York, 1989.

[9] Samuel M. Selby, editor. Standard Mathematical Tables. CRC Press, Inc., Cleveland, Ohio, 23 edition, 1975. 


\section{Acknowledgements}

The work described in this report was performed by the National Institute of Standards and Technology for the Naval Research Laboratory. The work was funded by the Office of Naval Research, Code 334, under the Damage Control Task of the FY98 BA2 Surface Ship Hull, Mechanical, and Electrical Technology Program (PE0602121N). 


\section{A Implementation of an Inter-compartment heat transfer algorithm.}

\section{A.1 Data Structures}

The required data structurcs required to implement inter-compartment heat transfer are listed below.

ihpart

ihwall

iside

IPARTINFOH(ihpart,j)

IPARTINFOV(ivpart,j)

IRMPART(iroom,j)

ivpart

ivstrip

MXHPART

MXVPART

NVPART

NHPART
An index used to specify the horizontal partition number.

An index used to specify whether the surface is a ceiling (ihwall=1) or a floor (ihwall=2).

An index used by the second component of the flux arrays; iside $=1$ corresponds to the front, while iside $=2$ corresponds to the back of the partition.

This integer array gives integer info about horizontal (floor and ceiling) partitions $(\mathrm{j}=1$ indicates the room number associated with side one of the partition, $\mathrm{j}=2$ indicates the room number associated with side two).

This integer array gives integer info about vertical (the walls) partitions (again $\mathrm{j}=1$ indicates the room number associated with side one of the partition, $j=2$ indicates the room number associated with side two).

The partitions associated with each room. The index values $j=1$ to $j=6$ give the partition numbers associated with the iroom'th room.

An index used to specify the vertical partition number.

An index used to specify the strip number on a vertical partition (a wall).

The number of horizontal partitions dimensioned in CFAST (equal to two times the number of rooms).

The number of vertical partitions dimensioned in CFAST (equal to four times the number of rooms).

The actual number of vertical partitions used in a particular CFAST run.

The actual number of horizontal partitions used in a particular CFAST run. the number of rooms). 
UFLUXCH(ihwall,ihpart,iside) Convective fluxes at the front and back of cach horizontal partition.

UFLUXCV(ivstrip,ivpart,iside) Convective fluxes at the front and back of each vertical partition strip.

UFLUXRH(ihwall,ihpart,iside) Radiative fluxes at the front and back of each horizontal partition.

UFLUXRV(ivstrip,ivpart,iside) Radiative fluxes at the front and back of each vertical partition strip.

UFLUXTH(ihwall,ihpart,iside) Total (radiative plus convective) fluxes at the front and back of each horizontal partition.

UFLUXTV(ivstrip,ivpart,iside) Total (radiative plus convective) fluxes at the front and back of each vertical partition strip.

UTEMPH(NWALLX,MSTRIP,ihpart) Temperature profile in horizontal wall partitions.

UTEMPV(NWALLX,MSTRIP,ivpart) Temperature profile in vertical wall partitions.

\section{A.2 Algorithms}

The algorithm for computing inter-compartment heat transfer may be summarized by

1. Assume an environment provided by DASSL, i.e. interior gas temperatures for each compartment and surface partition temperatures.

2. Given this environment compute radiative and convective fluxes for each partition strip using the algorithms described in either sections B.2 or 3.3. Store the fluxes in UFLUXTII for the floor and cciling and UFLUXTV for the four vertical walls.

3. Update the temperature profile within each partition using equation (17) if $1 \mathrm{D}$ heat conduction is modeled or both equations (17) and (18) if 2D heat conduction is modeled. Compute the "Fourier" residual $q^{\prime \prime}+K \frac{\partial u}{\partial x}$ for each partition strip.

DASSL provides a new environment in step 1. until the "Fourier" residual is reduced to essentially zero in step 3.

\section{B Implementation}

\section{B.1 Data Structures}

AMAT(NPAN,NPAN) coefficient matrix on left hand side of the net radiation equation 


$\begin{array}{ll}\text { AREA(NPAN) } & \begin{array}{l}\text { Area of each panel } \\ \text { coefficient matrix on right hand side of the net radiation equa- } \\ \text { tion }\end{array} \\ \text { BMAT(NPAN,NPAN) } & \begin{array}{l}\text { matrix of configuration factors } \\ \text { Number of strips in each vertical wall surface }\end{array} \\ \text { MSTRIP } & \begin{array}{l}\text { Total number of wall panels in a compartment (equal to 4*MSTRIP+2) } \\ \text { NPAN }\end{array} \\ \text { SOLF(NFIRE,NPAN) } & \text { Solid angle from each fire to each panel } \\ \text { TAUL(NPAN,NPAN) } & \text { Lower layer transmissivity between wall panels. } \\ \text { TAUU(NPAN,NPAN) } & \text { Upper layer transmissivity between wall panels. } \\ \text { TAUFL(NFIRE,NPAN) } & \text { Lower layer transmissivity between fires and wall panels. } \\ \text { TAUFU(NFIRE,NPAN) } & \text { Upper layer transmissivity between fires and wall panels. } \\ \text { TPAN(NPAN) } & \text { Surface temperature of each panel } \\ \text { XSOLN(NPAN) } & \text { Stores the solution to the net radiation equation }\end{array}$

\section{B.2 Algorithms}

1. Assume that the Temperature, $T$, emissivity, $\varepsilon$, and area, $A$, of each panel are given and stored in TPAN, EMIS and AREA.

2. Compute Configuration Factors (this only needs to be done once for each room)

(a) Compute configuration factor subset given by equations (29) to (33) and store results in appropriate columns of FIG.

(b) Compute the rest of FIG using equations (36) to (49)

3. Compute the net flux striking each wall panel

(a) Compute gas transmissivities

For each panel $j=1$ to $4 M+2$ and for each panel $k=j+1$ to $4 M+2$

i. Compute $L, L^{L}, L^{U}$ using equations (50), (51) and (52)

ii. Compute $\tau_{j-k}^{U}$ using equations (53) to (62). Store the result into TAUU.

iii. Compute $\tau_{j-k}^{L}$ using equations (64) to (73). Store the result into TAUL.

(b) Compute fire transmissivities For each fire $f$ and for each panel $k=1$ to $4 M+2$

i. Compute $L, L^{L}, L^{U}$ using equations (50), (51) and (52) 
ii. Compute $\tau_{j-k}^{\text {fire } U}, \tau_{j-k}^{\text {fire } L}$ using equations (75) and (76). Store the result into TAUFU and TAUFL.

(c) Compute fire solid angles using (87). Store the result into SOLF.

(d) Compute the flux from each fire $f$ to each panel $k$ using equation (89). Store the result into CGASFIRE.

(e) Compute the flux from each gas layer to each panel $k$ using equations (90) and (91). Accumulate the results into CGASFIRE.

(f) Compute matrices and vectors in the net radiation equation .

i. Compute $\operatorname{AMAT}(\mathrm{J}, \mathrm{K})=A_{J K}$ using equation (21).

ii. Compute BMAT(J,K) $=B_{J K}$ using equation (22).

iii. Compute $\mathrm{B}(\mathrm{K})=b_{K}$ using equation (23).

(g) Solve $A x=b$. The net flux striking each panel $k$ is given by $x_{k} \varepsilon_{k}$.

4. Compute the radiant heat absorbed by each gas layer

(a) compute QOUT, the total radiant flux emitted by a panel $j$ towards a panel $k$ using equation (92).

(b) compute the radiant heat absorbed by the lower and upper layers due to wall emission using equations (93) and (94).

(c) compute the radiant heat absorbed by the lower and upper layers due to gas layer emission using equations (97) and (98).

(d) compute the radiant heat absorbed by the lower and upper layers due to fires using equations (99) and (100). 


\begin{tabular}{|lr|}
\hline $\begin{array}{lr}\text { NIST-114 } \\
\text { (REV. 6-93) }\end{array}$ & U.S. DEPARTMENT OF COMMERCE \\
ADMAN 4.09 & NATIONAL INSTITUTE OF STANDARDS AND TECHNOLOGY \\
& MANUSCRIPT REVIEW AND APPROVAL \\
\cline { 2 - 3 } &
\end{tabular}

\begin{tabular}{|c|c|}
\hline (ERB USE ONL & \\
\hline ERB CONTROL NUMBER / & DIVISION \\
\hline $\begin{array}{l}\text { PUBLCATION REPORT NUMBER } \\
\text { NISTIR } 6190\end{array}$ & CAT \\
\hline
\end{tabular}

INSTRUCTIONS: ATTACH ORIGINAL OF THIS FORM TO ONE (1) COPY OF MANUSCRIPT AND SEND TO THE APPROPRIATE EDITORIAL REVIEW BOARD

PLALTARTON DATE

NUMBER PRINTED PAGES

TITLE AND SUBTITLE (CITE IN FULL)

A Method for Computing Heat Transfer Between Connected Compartments in a Zone Fire Model

\begin{tabular}{|l|l|l|}
\hline CONTRACT OR GRANT NUMBER & TYPE OF REPORT AND/OR PERIOD COVERED \\
\hline AUTHOR(S) (LAST NAME, FIRST INITIAL, SECOND INITIAL) & $\begin{array}{l}\text { PERFORMING ORGANIZATION (CHECK (X) ONE BOX) } \\
\text { Forney, Glenn P. and MOSS, William F. }\end{array}$ & $\begin{array}{l}\text { NIST/GAITHERSBURG } \\
\text { NIST/BOULDER } \\
\text { JILA/BOULDER }\end{array}$ \\
\hline
\end{tabular}

LABORATORY AND DIVISION NAMES (FIRST NIST AUTHOR ONLY)

Building and Fire Research Lahoratory, Fire Safety Engineering Division SPONSORING ORGANIZATION NAME AND COMPLETE ADDRESS (STREET, CITY, STATE, ZIP)

Naval Research Laboratory

PROPOSED FOR NIST PUBLICATION

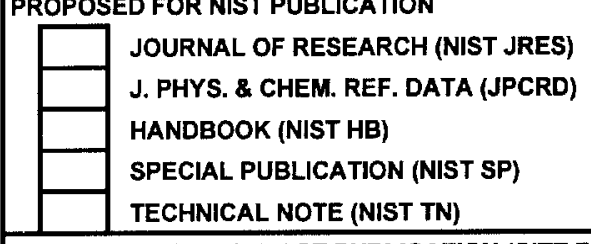

PROPOSED FOR NON-NIST PUBLICATION (CITE FULLY)

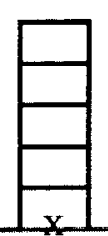

$x$
MONOGRAPH (NIST MN)

NATL. STD. REF. DATA SERIES (NIST NSRDS)

FEDERAL INF. PROCESS. STDS. (NIST FIPS)

LIST OF PUBLICATIONS (NIST LP)

NIST INTERAGENCYIINTERNAL REPORT (NISTIR)

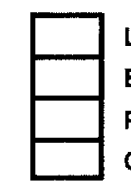

LETTER CIRCULAR BUILDING SCIENCE SERIES PRODUCT STANDARDS OTHER

\begin{tabular}{l|l|l|l|}
\hline & U.S. & FOREIGN & PUBLISHING MEDIUM
\end{tabular}

PAPER

DISKETTE (SPECIFY)

OTHER (SPECIFY)

SUPPLEMENTARY NOTES

ABSTRACT (A2000CHARACTERORLESSFACTUAL SUMMARY OF MOST SIGNFCANT NFORMATION FDOCUMENTNCLUDESASIGN FCANTERUOGRAPHYORLIERATURESURVEY, CIEITHERE SPELL OUT ACRONYMS ON FIRST REFERENCE.) (CONTINUE ON SEPARATE PAGE, IF NECESSARY.)

This note describes a method for calculating conductive heat transfer between connected compartments in a zone fire model. The conduction problem is solved by dividing a compartment into six partitions corresponding to the floor and ceiling and the front, right, back and left walls. The wall partitions are divided into several equally sized horizontal slabs or strips in order to approximate the vertical wall temperature

profile. Radiative and convective flux boundary conditions are computed for the front and back of each partition/strip.

Some of the difficulties encountered when computing radiation exchange for large numbers of wall surfaces are alleviated by taking advantage of symmetry and the numerical characteristics of the linear system of equations that are solved. All strips have the same height. Strips on opposite walls have the same width. Strips do not change size with time. Using this information, the number of necessary configuration factor and transmissivity factors are reduced substantially. For wall surfaces that are nearly black, emissivities close to one, the system of equations to be solved (the net radiation

KEY WORDS (MAXIMUM OF 9; 28 CHARACTERS AND SPACES EACH; SEPARATE WITH SEMICOLONS; ALPHABETIC ORDER; CAPITALIZE ONLY PROPER NAMES)

heat transfer; radiation modeling; radiative heat transfer; zone models

AVAILABILITY

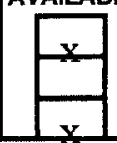

UNLIMITED ORDER FROM SUPERINTENDENT OF DOCUMENTS, U.S. GPO, WASHINGTON, DC 20402 ORDER FROM NTIS, SPRINGFIELD, VA 22161
NOTE TO AUTHOR(S): IF YOU DO NOT WISH THIS MANUSCRIPT ANNOUNCED BEFORE PUBLICATION, PLEASE CHECK HERE. 